\title{
High Performance Thin-Layer Chromatography of Plant Ecdysteroids Coupled with Desorption Electrospray Ionisation-Ion Mobility-Time of Flight High Resolution Mass Spectrometry (HPTLC/DESI/IM/ToFMS)
}

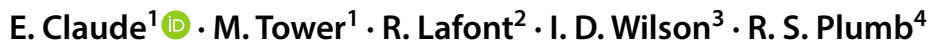

Received: 27 January 2020 / Revised: 18 May 2020 / Accepted: 29 May 2020 / Published online: 7 June 2020

(c) The Author(s) 2020

\begin{abstract}
The use of high performance thin-layer chromatography (HPTLC) in combination with high resolution time of flight mass spectrometry (MS) for the detection, identification and imaging (HPTLC/MSI) of ecdysteroids (insect moulting hormones) present in a number of plant extracts obtained from members of the Silene family is demonstrated. DESI is shown to be a convenient method for the recovery of these polar polyhydroxylated steroids from the silica gel of the HPTLC plate for subsequent MS detection and imaging. The incorporation of an ion mobility separation (IMS) to the system to give HPTLC/IMS/ MS provided additional drift time data which enabled more confident identification. Using HPTLC/DESI/IMS/MS, a range of ecdysteroids were detected and characterized in extracts of S. otitis, S nutans, S. maritime, S. viridiflora and S. fimbriata.
\end{abstract}

Keywords HPTLC-MS · Ion mobility $\cdot$ DESI $\cdot$ Ecdysteroids $\cdot$ Imaging MS

\section{Introduction}

The ecdysteroids form a group of polar, polyhydroxylated, steroids that are involved in arthropod development, particularly in regard to moulting (and indeed these compounds are often referred to as moulting hormones). The first of these compounds to be isolated was ecdysone ((22R)-2 $\beta, 3 \beta, 14 \alpha, 22,25$-pentahydroxy- $5 \beta$-cholest-7-en6-one) in 1954 by Butenant and Karlson [1]. Since then some 517 ecdysteroids have been described [2], many derived from plants, where they probably act as defence compounds against insects. More recently these secondary

E. Claude

emmanuelle_claude@waters.com

$\triangle$ I. D. Wilson

i.wilson@imperial.ac.uk

1 Waters Corporation, Stamford Rd, Wilmslow SK9 4AX, UK

2 Sorbonne Université, IBPS-BIOSIPE, Campus Pierre et Marie Curie, 75005 Paris, France

3 Division of Systems Medicine, Department of Metabolism, Digestion and Reproduction, Imperial College London, Exhibition Road, South Kensington, London SW7 2AZ, UK

4 Scientific Operations, Waters Corporation, Milford, MA 01757, USA plant metabolites, also termed phytoecdysteroids, have been also assessed for pharmacological properties such as e.g., anabolic effects in humans [3]. When searching for phytoecdysteroids TLC provided a proven, robust and readily available method for screening extracts, of varying degrees of purity, but identification remains a problem. For the identification of ecdysteroids, and indeed many other secondary plant products, MS-based techniques represent a mainstay for their spectrometric characterisation. Often MS for the detection and characterization of compounds in complex mixtures is performed in conjunction with hyphenation to a separation technique. Whilst hyphenation has most commonly been to column-based techniques (e.g., GC, LC CE etc.) the application of TLC/MS has also been an area of some interest for many years [4]. Various methods, either via "direct" (e.g., MALDI/ToFMS-based [5, 6]), or using more specialized TLC/MS interfaces [e.g., 7, 8], amongst others, have been described. In previous studies, we have used off-line fast atom bombardment (FAB) MS to analyse ecdysteroids contained in plant extracts directly from the silica of HPTLC plates. In these applications the stationary phase for the zones of interest was removed from the plate and mixed (mulled) with glycerol and DMSO to form a paste before being placed directly on the FAB target [9]. However, whilst this manual process was effective, it was time consuming and not quantitative, and much spatial 
information was lost. The development of desorption electrospray ionisation techniques (DESI) for MS, that are well suited to both targeted "spot" analysis and TLC plate imaging, e.g. for lipids [10] and alkaloids [11], has encouraged us to reinvestigate the application of HPTLC/MS directly by DESI for the analysis and identification of ecdysteroids in plant extracts. We have also previously investigated the use of TLC with DESI in combination with ion mobility (IM) [12]. The use of an ion mobility separation (IMS) provides a fast-analytical technique that separates gas-phase ions on the basis of their differential mobility. This differential mobility is a product of their size, shape, $\mathrm{m} / \mathrm{z}$ and charge in a buffer gas under the influence of an applied electric field and our initial investigation used a dedicated TLC plate interface applied to the DESI/IMS/MS of pharmaceuticals [12]. More recently, the use of TLC/IMS alone has been described for the offline and online analysis of TLC plates for two component mixtures of morphine-papaverine and acridine-papaverine [13] but, while valuable, the identification potential of IMS alone, even when coupled to a TLC separation, for complex mixtures is limited. Combining complementary analytical techniques such as HPTLC, DESI, MS and IM into a single method offers advantages such as reduced sample preparation, retention of spatial information, increased peak capacity, improved MS-spectra and greater confidence in identification. Here we describe the use of DESI/ MS imaging (MSI) and DESI/IMS/MSI for the analysis of ecdysteroid-containing plant extracts, separated by HPTLC, as an example of its use for more complex mixtures.

\section{Experimental}

\section{Chemicals and Reagents}

Chloroform and ethanol were obtained from Sigma Aldrich (Dorset, UK). The ecdysteroids used as standards in this work were obtained as gifts from a variety of sources and were used as supplied.

Samples: standard ecdysteroids were dissolved in methanol at a concentration of ca. $1 \mathrm{mg} / \mathrm{ml}$ and $1 \mu \mathrm{l}$ of each was applied to the plate. The five methanolic extracts from four members of the Silene genus, namely $S$. nutans (two different extracts, SN1 and SN2), S. otites (SO1), S. fimbriata (SF) and $S$. viridiflora (SV1) were used in these studies prepared as described elsewhere [9].

Chromatography: Chromatography was performed by HPTLC on silica gel, on both ecdysteroid standards (aluminium backed HPTLC plates $5 \times 7.5 \mathrm{~cm}$ ) and plant extracts (glass backed silica gel HPTLC plates, $10 \times 10 \mathrm{~cm}$ ) incorporating a fluorescent indicator (E. Merck, Darmstadt, Germany). Plate development was performed using glass TLC tanks, in a fume hood, with chloroform: ethanol 4:1 (v/v) as the developing solvent. Samples were applied to the plates manually using $1 \mu \mathrm{l}$ glass capillaries (Drummond Scientific Company, Broomall, Pa, USA). The quality of the separations achieved was assessed prior to further MS-based analysis by visualizing the plates under a UV lamp at $254 \mathrm{~nm}$ and, if suitable, they were then taken for DESI.

\section{DESI-MS Imaging}

Experiments were performed using a SYNAPT XS High Definition MS (HDMS) system (Waters Corporation, Manchester, UK) operated in sensitivity mode $(25,000 \mathrm{FWHM}$ at $\mathrm{m} / \mathrm{z}, 500$ ) and equipped with a two-dimensional DESI stage from Prosolia (Indianapolis, IN, USA). A methanol/water $\left(\mathrm{MeOH} / \mathrm{H}_{2} \mathrm{O}\right)$ 98:2 (v/v) solvent composition was used at a flow rate of $2 \mu \mathrm{l} / \mathrm{min}$ with a nebulizing gas spray of nitrogen at a gas pressure of 5 bar. A voltage of $4 \mathrm{kV}$ was applied to the spray.

DESI/MSI experiments were acquired in positive mode from $m / z, 100-1200$ with ion mobility separation (IMS) key settings of trap DC bias 50, IMS wave velocity ramp starting at $1,000 \mathrm{~m} / \mathrm{s}$ and ending at $250 \mathrm{~m} / \mathrm{s}$ and transfer wave velocity of $175 \mathrm{~m} / \mathrm{s}$. The pixel dimensions were $200 \mu \mathrm{m}$ in both the $X$ and $Y$ dimensions.

\section{Data Processing}

Acquisition setup, processing and visualisation of imaging data were performed using High Definition Imaging (HDI) 1.5 (Waters Corporation, Manchester, UK). Data were acquired and mined using MassLynx version 4.2 (Waters Corporation, Manchester, UK). Further data mining was carried out using DriftScope 2.9 (Waters Corporation, Manchester, UK) to visualise the IMS dimension of the data. Datasets were re-arranged by drift time (either bins or milliseconds) to evaluate the different drift time (Dt) of the compounds of interest. Also, using DriftScope, it was possible to extract distinct regions in the $\mathrm{m} / \mathrm{z}$ vs. Dt 2D-plot corresponding to different classes of molecules present on the surface of the samples.

\section{Results and Discussion}

Initial experiments to determine the suitability of DESI/ MSI were performed with standards chromatographed on aluminium backed silica gel HPTLC plates, using chloroform-ethanol 4:1 (v/v). This solvent system was chosen as it is widely used for the separation of ecdysteroids on silica gel. After drying, the developed plates were taken for analysis by DESI/MSI and then DESI/IMS/MSI as described below. Whilst DESI/MS of the ecdysteroids was found to be 
possible using either aluminium or glass backed plates the result provided below are for the latter.

\section{DESI/MSI and TLC/DESI/IMS/MS of Ecdysteroid Standards}

Five ecdysteroid standards (ponasterone A, ecdysone, kaladasterone, inokosterone and 20-hydroxyecdysone) were spotted onto a Prosolia Teflon coated glass slide to ensure that these molecules could be analysed directly by DESI/ MS. All standards were detected as sodiated species by
DESI/MS, with little or no presence of the protonated or potassiated species as seen in Fig. 1a. Drift times (Dt) of the sodiated molecular ion for each standard are plotted in Fig. $1 \mathrm{~b}$ and summarised in Table 1. Ponasterone A and ecdysone are isobaric molecules and were detected as $\mathrm{m} / \mathrm{z}$ 487.30 but with $\mathrm{Dt}=5.1 \mathrm{~ms}$ and $\mathrm{Dt}=5.37 \mathrm{~ms}$, respectively. Kaladasterone was detected as $m / z 501.28$ and $\mathrm{Dt}=5.30 \mathrm{~ms}$ and inokosterone and 20-hydroxyecdysone (which are also isobaric) were detected as sharing both the same mass $(\mathrm{m} / \mathrm{z}$ 503.30) and the same drift times $(\mathrm{Dt}=5.17 \mathrm{~ms})$ which makes these two species difficult to differentiate. No fragmentation

(b) Drift time chromatogram
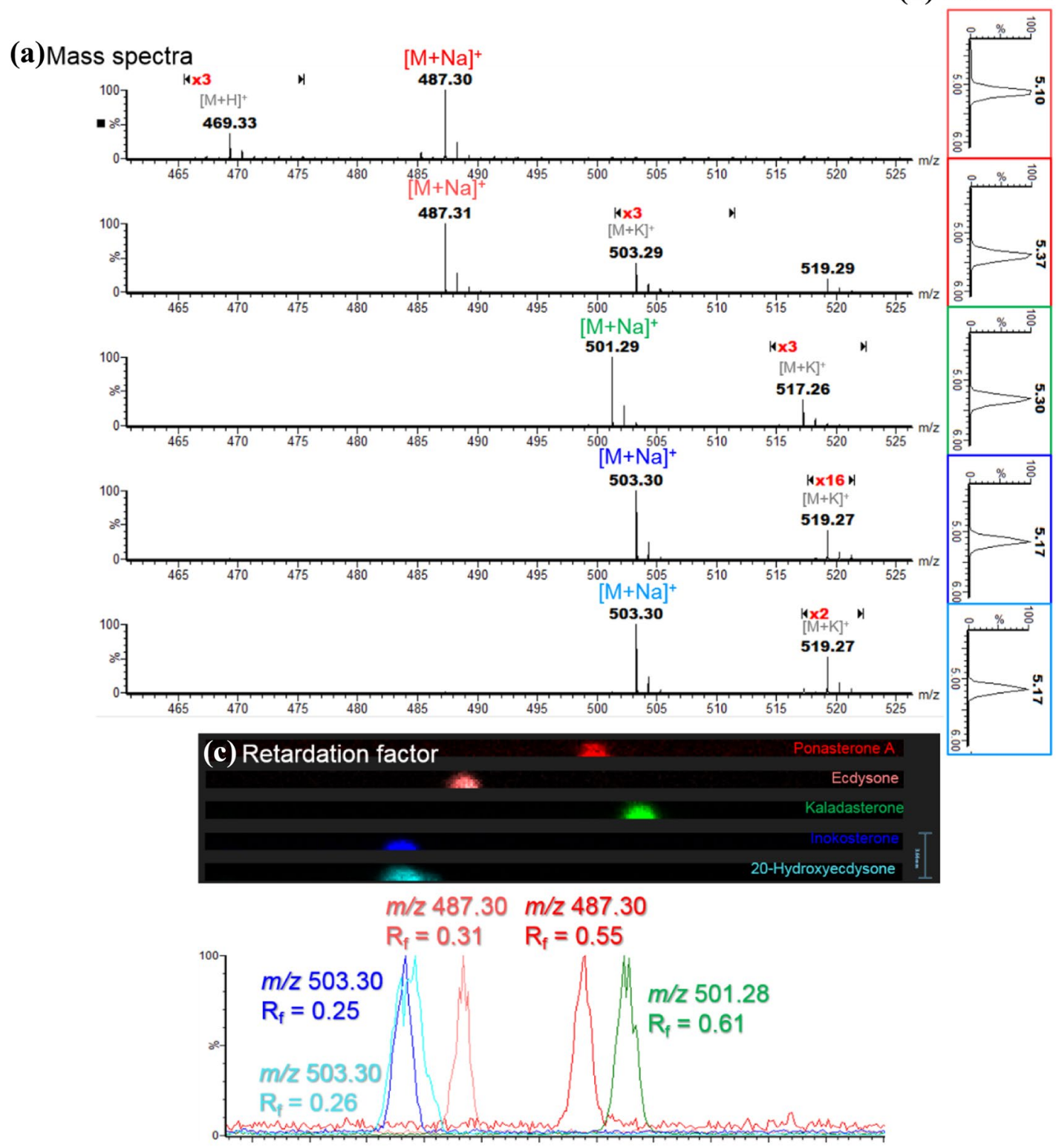

Fig. 1 a MS spectra of each ecdysteroid standard (ponasterone A, ecdysone, kaladasterone, inokosterone and 20-hydroxyecdysone) at $\mathbf{b}$ the specific drift time (Dt) of the sodiated molecular ion. $\mathbf{c}$ Ion images of the five ecdysteroid standards chromatographed by TLC and directly analysed by DESI MSI, with the overlay of the extracted ion chromatograms of the ecdysteroid standards shown below 
Table 1 Summary table reporting the $m / z$ of the sodiated molecular ion, retardation factor $\left(R_{\mathrm{f}}\right)$ and drift time (Dt) for the five ecdysteroid standards (ponasterone A, ecdysone, kaladasterone, inokosterone and 20-hydroxyecdysone) analysed by HPTLC DESI IMS/MS

\begin{tabular}{llll}
\hline & {$[\mathrm{M}+\mathrm{Na}]^{+}$} & $\begin{array}{l}\text { Retardation } \\
\text { factor }(R \mathrm{f})\end{array}$ & $\begin{array}{l}\text { Drift time } \\
(\mathrm{Dt})(\mathrm{ms})\end{array}$ \\
\hline Ecdysone & 487.3036 & 0.31 & 5.1 \\
Ponasterone A & 487.3036 & 0.55 & 5.37 \\
Kaladasterone & 501.2828 & 0.61 & 5.3 \\
Inokosterone & 503.2985 & 0.25 & 5.17 \\
20-Hydroxyecdysone & 503.2985 & 0.26 & 5.17 \\
\hline
\end{tabular}

was detected for any of the ecdysteroid standards when subjected to either low energy or elevated collision energy CID fragmentation. Following the successful detection of each of the standards they were then chromatographed individually on a glass-backed silica gel HPTLC plate, which was then directly mounted onto the DESI stage for imaging. DESI/ MSI analysis was then attempted with no additional sample preparation.

From Fig. 1c, it can be observed that the isobaric species ponasterone A and ecdysone have different $R_{\mathrm{f}}$ values $\left(R_{\mathrm{f}} 0.55\right.$ and $R_{\mathrm{f}} 0.31$, respectively). However, the second pair of isobaric standards, inokosterone and 20-hydroxyecdysone, were not resolved by TLC as they shared a similar $R_{\mathrm{f}}$ of ca. 0.25 .

\section{Analysis of Plant Extracts by TLC/DESI/MSI}

The methanolic S. fimbriata extract was seen to contain a number of components that had been separated by TLC and detected by both UV and DESI/MS (Fig. 2). The detection of these compounds by UV is possible because the ecdysteroids as a class all contain a 7-en-6-one in the steroidal B ring. This en-one provides a chromophore that confers a strong UV absorbtion at $243 \mathrm{~nm}$, making ecdysteroids easily visible on TLC plate containing a fluorescent indicator absorbing at $254 \mathrm{~nm}$. Thus, examination of the TLC plate under UV light $(\lambda=254 \mathrm{~nm})$ revealed a number of intensely absorbing spots with the strongest at an $R_{\mathrm{f}}$ of 0.28 . This spot was also detected by DESI-IMS-MS, with the highest signal intensity at $\mathrm{m} / \mathrm{z}$ 503.29. Based on mass accuracy and $R_{\mathrm{f}}$, it could therefore be putatively identified as either inokosterone or 20-hydroxyecdysone. A second potential ecdysteroid from the extract was putatively identified as ponasterone A with $m / z, 487.3$ and $R_{\mathrm{f}} 0.55$.

Interestingly, as shown in Fig. 2, three TLC spots were detected for $m / z 519.29\left(\mathrm{C}_{27} \mathrm{H}_{44} \mathrm{O}_{8}, \mathrm{Na}^{+}\right)$, at $\mathrm{R}_{\mathrm{f}}$ 's of 0.09 , 0.23 and 0.39 , corresponding to different isomeric ecdysteroids. The latter is most probably polypodine $\mathrm{B}$, which is a very common phytoecdysteroid in plants [14], and which has previously been found in Silene extracts. However, according to the Ecdysteroids Database (https://ecdybase. org), at least 24 different isomers for $\mathrm{C}_{27} \mathrm{H}_{44} \mathrm{O}_{8}, \mathrm{Na}^{+}$have been reported in the literature, as will be discussed further in the next section.

As can be seen from the DESI imaging trace for the S. fimbriata (SF) extract, at least ten spots were detected with a significant level of intensity which makes the fingerprint more complex and informative compared to the $\mathrm{UV}_{254}$ absorbance TLC fingerprint alone. However, as a number of these spots lack a UV chromophore absorbing at $254 \mathrm{~nm}$ they are likely not ecdysteroid-related suggesting that they are co-extracted "impurities". (a)

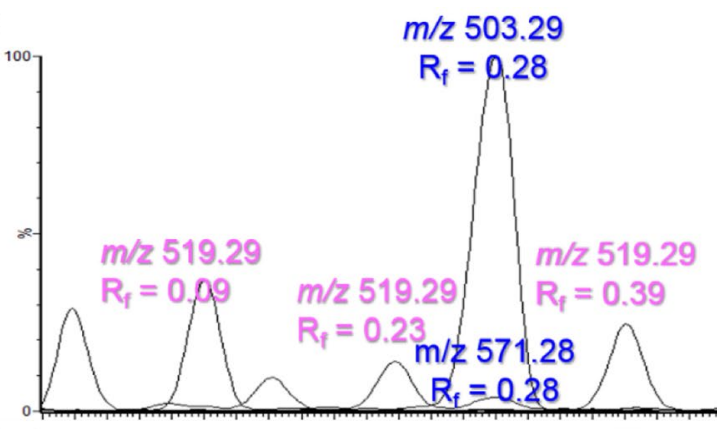

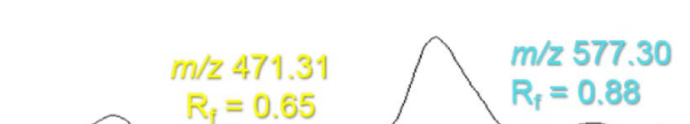

(b)

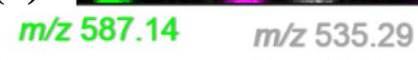

(c)
$R_{\mathrm{f}}=0.14$ $m / z 487.30$

$R_{\mathrm{f}}=0.55$ $m / 2467.30$

$R_{f}=0.78$ $m / z 595.38$

$R_{f}=0.93$

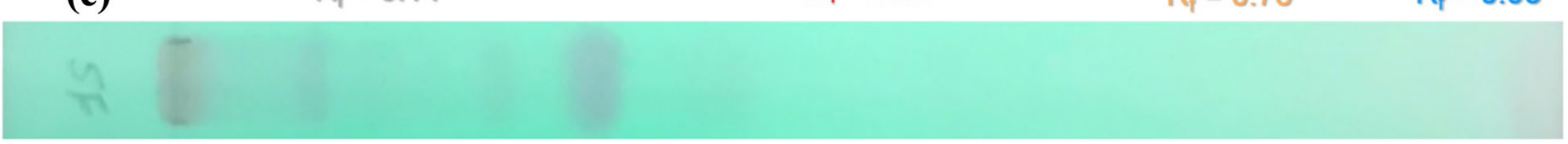

Fig. 2 a Overlay of the DESI-extracted chromatograms of the different molecules separated on the TLC plate from extract $S$. fimbriata with the $\mathrm{m} / \mathrm{z}$ and $\mathrm{Rf}$ for each of the peak, $\mathbf{b}$ overlay of the ion images from the TLC DESI-MS imaging experiment, c UV absorbtion image of the TLC plate before DESI acquisition 
Four other plant extracts were subjected to TLC separation along with the SF extract: one from $S$, otites (SO1), one from $S$. viridiflora (SV1) and two obtained from different samples of S. nutans (SN1 and SN2). All of these extracts contained 20-hydroxyecdysone (or one of its isomers) $(\mathrm{m} / \mathrm{z}$ 503.29, $R_{\mathrm{f}}$ 0.28). Furthermore, as evident from Fig. $3, \mathrm{~m} / \mathrm{z}$ $487.3, R_{\mathrm{f}} 0.54$ (potentially ecdysone or ponasterone $\mathrm{A}$ ) and a number of ions at $m / z 519.3$, with $R_{\mathrm{f}}$ values of $0.09,0.23$ and 0.39 , respectively were detected in some, but not all, of the extracts, albeit at different levels of intensity. One of these, with the $R_{\mathrm{f}}$ of 0.39 is probably polypodine B which is commonly found in specimens of this genus [14] whilst candidates for the other ions sharing the ions at $\mathrm{m} / \mathrm{z} 519.3$ include integristerone A, abutasterone and 20,26-dihydroxyecdysone (the latter in particular (and its derivatives) has been found in these species, e.g. see [15]). In addition, some of the presumed ecdysteroids were detected in only one extract, i.e. $m / z 467.30\left(R_{\mathrm{f}} 0.77\right)$, was only found in the S. fimbriata extract, whilst $m / z 471.31\left(R_{\mathrm{f}} 0.65\right)$ was only present in the $S$, otitis extract, and $m / z 530.42\left(R_{\mathrm{f}} 0.68\right)$ in one of the $S$. nutans extracts. The ecdysteroid with $m / z 471.31\left(R_{\mathrm{f}} 0.65\right)$ is most likely to be 2-deoxyecdysone, which has previously been shown to be a major compound for S. otites [9].
As is clear, for all of these samples, more analytes were detected in the DESI MSI traces compared to the equivalent TLC UV absorbtion fingerprints, allowing a better idea of purity with respect to the extracted ecdysteroid fraction to be made.

\section{Benefits of IMS for the analysis of the plant extracts by TLC/DESI/IMS/MSI}

As noted in the previous section, under these conditions DESI provides good $\mathrm{M}+\mathrm{Na}$ ions but little in the way of fragmentation was obtained that would aid structure elucidation. One problem is that, in the case of the ions with a $\mathrm{m} / \mathrm{z}$ of 519.29 there are potentially 24 known isobaric ecdysteroids, including polypodine $\mathrm{B}$. With the addition of the IMS dimension to the HPTLC/DESI/MSI system, a combination of $R_{\mathrm{f}}$, Dt and $\mathrm{m} / \mathrm{z}$, is obtained affording a "3D" separation and detection system for isobaric ecdysteroids enabling both $R_{\mathrm{f}}$ and Dt to be used to distinguish these molecules when the MS data alone cannot. When a standard for polypodine B was analysed by DESI/IMS one ion mobility peak was detected in the extracted mobilogram for $m / z 519.29$ with a drift time of $5.43 \mathrm{~ms}$ (as seen in Fig. 4, $a_{1}$ ). In Fig. 4, $a_{2}$, the extracted mobilogram for

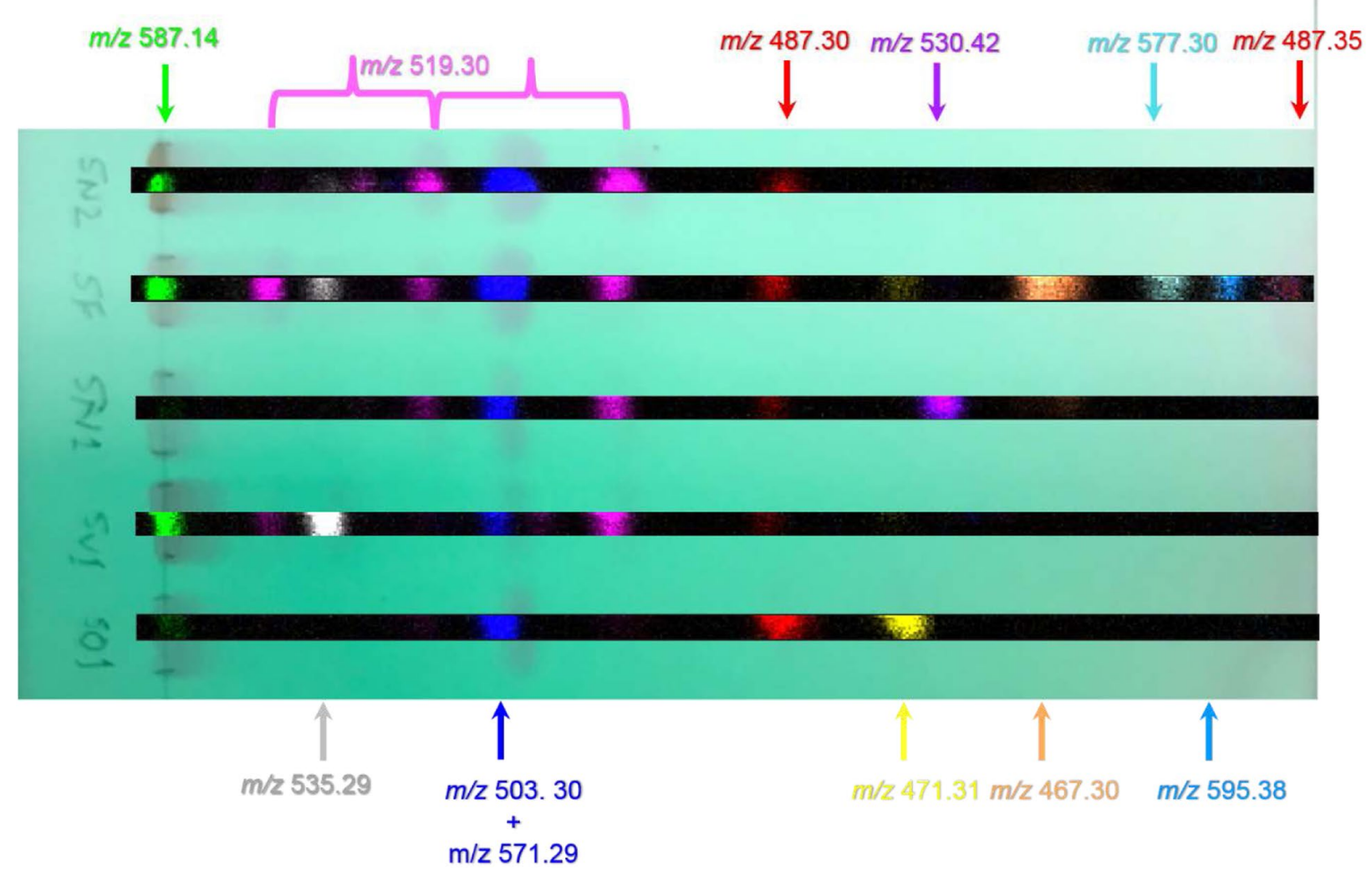

Fig. 3 Overlay of the UV-absorbing trace of the TLC plate before DESI acquisition with the ion images of the different colour coded spots of different $m / z$ for $S$, otitis (SO1), S. viridiflora (SV1), two $S$. nutans (SN1 and SN2) and the $S$. fimbriata plant extracts 


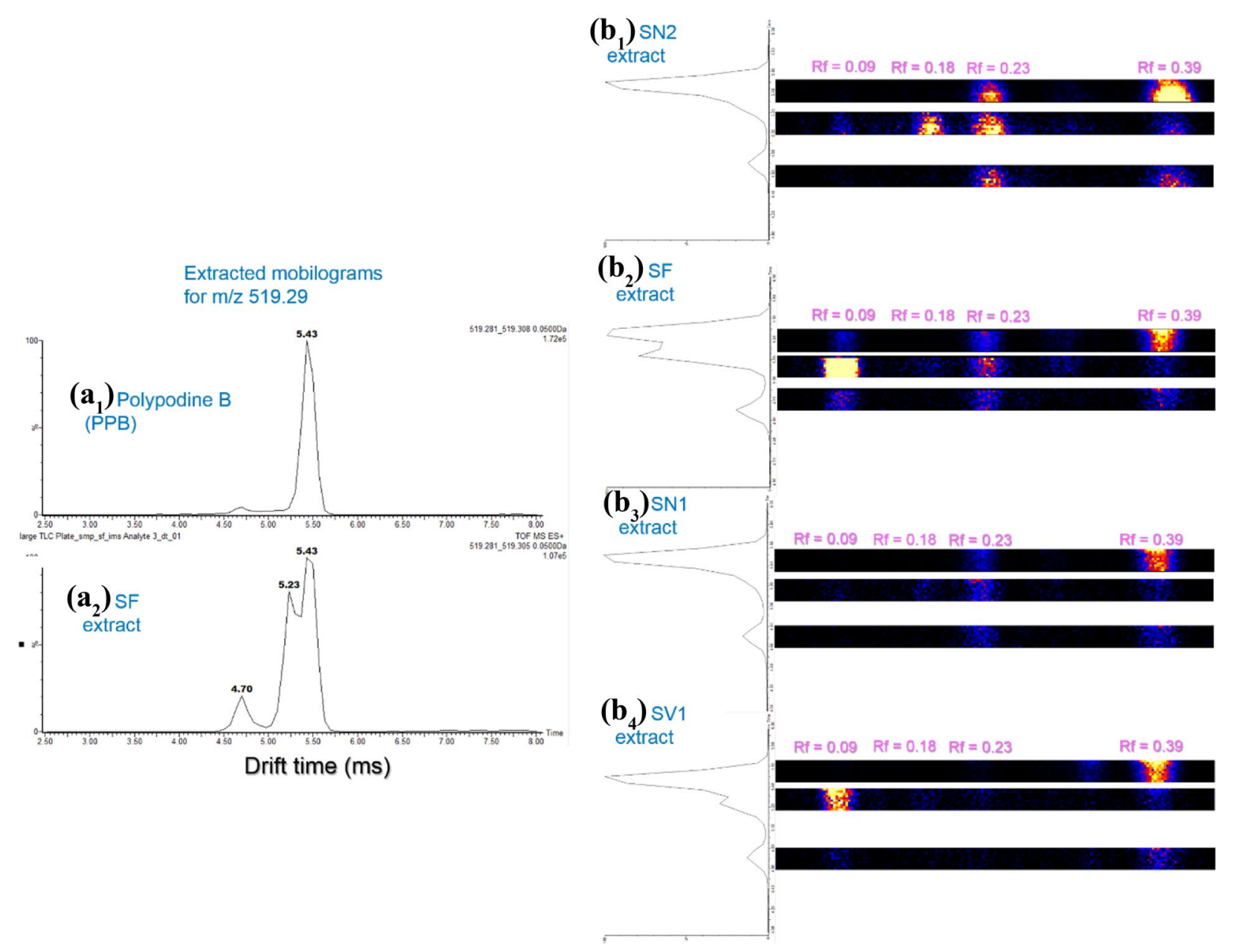

Fig. 4 a Extracted mobilograms of $\mathrm{m} / \mathrm{z} 519.29$ for $\mathbf{a}_{1}$ polypodine B (PPB) standard, $\mathbf{a}_{2} S$. fimbriata plant extract. Extracted mobilograms of $\mathrm{m} / \mathrm{z} 519.29$ combined with DESI IMS MSI trace for $\mathbf{b}_{1} S$. nutans

$m / z 519.29$ for $S$. fimbriata extract shows three peaks with Dt's of 4.7, 5.23 and $5.43 \mathrm{~ms}$, respectively. The peak with the greatest intensity was found to have at Dt of $5.43 \mathrm{~ms}$ and can be putatively identified as polypodine $B$ on the basis of both mass spectral and drift time data.

The extracted mobilograms for $m / z 519.29$ from $S$, otites (SO1), S. viridiflora (SV1) and S. nutans (SN1 and SN2) plant extracts are shown in Fig. $4 \mathrm{~b}$ along with the DESI/ IMS imaging trace for each specific drift time. As can be seen, all of these extracts have the most intense IMS peak as having a Dt of $5.43 \mathrm{~ms}$ and are also therefore most likely to correspond to polypodine $\mathrm{B}$, which would be consistent with published literature.

The two $S$. nutans extracts (SN1 and SN2) had the same IMS profile and DESI traces, showing eight different TLC spots sharing the three different drift times. In addition, the mobilograms for the $S$, otites (SO1) and S. viridiflora (SV1) samples shared an additional IMS peak, with a Dt of $5.23 \mathrm{~ms}$

S 2 (SN2), $\mathbf{b}_{2}$ S. fimbriata (SF), $\mathbf{b}_{3} S$. nutans $1(\mathrm{SN} 1)$ and $\mathbf{b}_{4} S$. viridiflora (SV1) plant extracts

and a $R_{\mathrm{f}}$ of 0.09 , that probably results from the same isomer being present in both extracts between the two plant extracts.

In future studies, the number of ecdysteroid standards for which IMS data have been collected will be increased to aid in identification, in combination with additional HPTLCderived $R_{\mathrm{f}}$ data.

\section{Conclusions}

These preliminary investigations show that the combination of DESI/MS coupled to the imaging of HPTLC plates represents an effective method for the preliminary screening of plant extracts for target compounds such as these ecdysteroids. The methodology avoids the need for either the removal of the stationary phase and its subsequent extraction, or specialized interfaces to elute the compounds of interest directly from the plate. In addition, due to the mechanism of DESI no sample preparation is required prior 
to imaging, unlike e.g., MALDI, where a matrix must be applied prior to analysis. DESI/MSI also produced richer images than provided by the UV data, giving more specific fingerprints for the plant samples and providing an indication of the prevalence of contaminants in the extracts. The addition of the IMS adds an additional, orthogonal, dimension to the overall separation and can enable co-migrating/ isobaric compounds to be resolved, therefore giving a more complete picture of all the molecules presents in a mixture. The differential mobility measurements obtained, can also aid identification.

Funding No external funding was used in conducting this study.

\section{Compliance with Ethical Standards}

Conflict of interest The authors declared no conflicts of interest with respect to the research, authorship, and/or publication of this article.

Open Access This article is licensed under a Creative Commons Attribution 4.0 International License, which permits use, sharing, adaptation, distribution and reproduction in any medium or format, as long as you give appropriate credit to the original author(s) and the source, provide a link to the Creative Commons licence, and indicate if changes were made. The images or other third party material in this article are included in the article's Creative Commons licence, unless indicated otherwise in a credit line to the material. If material is not included in the article's Creative Commons licence and your intended use is not permitted by statutory regulation or exceeds the permitted use, you will need to obtain permission directly from the copyright holder. To view a copy of this licence, visit http://creativecommons.org/licenses/by/4.0/.

\section{References}

1. Butenandt A, Karlson P (1954) Über die Isolierung eines Metamorphose-Hormons der Insekten in kristallisierter Form. Zeitschrift für Naturforschung B 9:389-391

2. Lafont R, Harmartha J, Marion-Poll F, Dinan, L, Wilson ID (2002 continuously updated) The Ecysone Handbook. https://ecdybase. org/

3. Isenmann E, Ambrosio G, Joseph JF, Mazzarino M, de la Torre X, Zimmer P, Kazlauskas R, Goebel C, Botrè F (2019) Ecdysteroids as non-conventional anabolic agent: performance enhancement by ecdysterone supplementation in humans. Arch Toxicol 93:1807-1816
4. Wilson ID (1999) The state of the art in thin-layer chromatography-mass spectrometry: a critical appraisal. J Chromatogr A 856:429-442

5. Griesinger H, Fuchs B, Süß R, Matheis KM, Schulz J, Schiller J (2014) Stationary phase thickness determines the quality of thinlayer chromatography/matrix-assisted laser desorption and ionization mass spectra of lipids. Anal Biochem 451:45-47

6. Kucherenko E, Kanateva A, Pirogov A, Kurganov A (2019) Recent advances in the preparation of adsorbent layers for thinlayer chromatography combined with matrix-assisted laser desorption/ionization mass-spectrometric detection. J Sep Sci 2:415-430

7. Oellig C, Schwack W (2012) Planar solid phase extraction cleanup for pesticide residue analysis in tea by liquid chromatographymass spectrometry. J Chromatogr A 1260:42-53

8. Morlock GE, Ristivojevica P, Chernetsova ES (2014) Combined multivariate data analysis of high-performance thin-layer chromatography fingerprints and direct analysis in real time mass spectra for profiling of natural products like propolis. J Chromatogr A 1328:104-112

9. Wilson ID, Lafont R, Wall P (1988) TLC of ecdysteroids with offline identification by fast atom bombardment mass spectrometry directly from the adsorbent. J Planar Chromatogr 1:357-359

10. Paglia G, Ifa DR, Wu C, Corso G, Cooks RG (2010) Desorption electrospray ionization mass spectrometry analysis of lipids after two-dimensional high-performance thin-layer chromatography partial separation. Anal Chem 82:1744-1750

11. Van Berkel GJ, Tomkins BA, Kertesz V (2007) Thin-layer chromatography/desorption electrospray ionization mass spectrometry: investigation of goldenseal alkaloids. Anal Chem 79:2778-3278

12. Harry EL, Reynolds JC, Bristow AWT, Wilson ID, Creaser CS (2009) Direct analysis of pharmaceutical formulations from nonbonded reversed-phase thin-layer chromatography plates by desorption electrospray ionisation ion mobility mass spectrometry. Rapid Commun Mass Spectrom 23:2597-2604

13. Ilbeigi V, Tabrizchi M (2015) Thin layer chromatography-ion mobility spectrometry (TLC-IMS). Anal Chem 87:464-469

14. Mamadalieva NZ, Zibareva LN, Lafont DLN, Saatov Z (2004) Phytoecdysteroids from the Silene genus. Chem Nat Comp 40:574-578

15. Toth, Simon A, Toth A, Kele G, Hunyadi Z, Bathori A (2008) 26-Hydroxylated Ecdysteroids from Silene viridiflora. J Nat Prod 71:1461-1463

Publisher's Note Springer Nature remains neutral with regard to jurisdictional claims in published maps and institutional affiliations. 